of the air, environmental surfaces, and water distribution system of a hospital that had known cases of aspergillosis. The purpose of the study was to determine other possible sources of infection.

Aspergillus species were found in the hospital water system. Significantly higher concentrations of airborne Aspergillus propagules were found in bathrooms, where water use was highest (2.95 colony-forming units $\left.[\mathrm{CFU}] / \mathrm{m}^{3}\right)$, than in patient rooms $\left(0.78 \mathrm{CFU} / \mathrm{m}^{3} ; P=.05\right)$ and in hallways $\left(0.61 \mathrm{CFU} / \mathrm{m}^{3} ; P=.03\right)$. A correlation was found between the rank orders of Aspergillus species recovered from hospital water and air. Water from tanks yielded higher counts of colony-forming units than did municipal water. An isolate of $A$. fumigatus recovered from a patient with aspergillosis was genotypically identical to an isolate recovered from the shower wall in the patient's room.

The authors concluded that in addition to the air, hospital water systems may be a source of nosocomial aspergillosis.

FROM: Anaissie EJ, Stratton SL, Dignani MC, et al. Pathogenic Aspergillus species recovered from a hospital water system: a 3-year prospective study. Clin Infect Dis 2002;34:780-789.

\section{Three Consecutive Outbreaks of Serratia marcescens in a Neonatal Intensive Care Unit}

Fleisch and colleagues from the University Hospital of Zurich, Switzerland, investigated an outbreak of Serratia marcescens in the neonatal intensive care unit (NICU) of this institution. S. marcescens infection was detected in four children transferred from the NICU to the University Children's Hospital (Zurich). All isolates showed identical banding patterns by pulsed-field gel electrophoresis. In a prevalence survey, 11 of 20 neonates were found to be colonized. $S$. marcescens was isolated from bottles of liquid theophylline. Despite replacement of these bottles, $S$. marcescens colonization was detected in additional patients.

Prospective collection of stool and gastric aspirate specimens revealed that colonization occurred in some infants within 24 hours after delivery. These isolates showed a different genotype. Cultures of milk from used milk bottles yielded $S$. marcescens. These isolates showed a third genotype. The method of reprocessing bottles was changed to thermal disinfection. In follow-up prevalence studies, 0 of 29 neonates were found to be colonized by $S$. marcescens.
In summary, three consecutive outbreaks caused by three genetically unrelated clones of $S$. marcescens were documented. Contaminated milk could be identified as the source of at least the third outbreak.

FROM: Fleisch F, Zimmermann-Baer U, Zbinden R, et al. Three consecutive outbreaks of Serratia marcescens in a neonatal intensive care unit. Clin Infect Dis 2002;34:767-773.

\section{MRSA Epidemic Clone in Argentina Involved in Nosocomial Infections}

Infections caused by methicillin-resistant Staphylococcus aureus (MRSA) are a major health concern worldwide for hospitalized patients. In addition, the incidence of community-acquired infection has risen continuously during the past few years. Some MRSA clones spread easier than others within the hospital environment and therefore are frequently implicated in outbreaks. Thus, the spread of a unique epidemic multiresistant clone, the so-called South American clone, is the main cause of nosocomial infections produced by this bacterium in Brazil and in some regions of Argentina, Chile, and Uruguay. Sola and colleagues from the Instituto de Salud Carlos III, Centro Nacional de Microbiologia, Madrid, Spain, describe the identification of a novel clone of MRSA that is involved in nosocomial infections and that shows a prevalence as high as that of the South American clone.

A total of 53 consecutive single-patient MRSA isolates were recovered during a 3-month period (May to July 1999) from six different hospitals ( 955 beds) in Cordoba. The isolates were initially typed according to the antibiotic resistance and phage susceptibility patterns, followed by genotyping using pulsed-field gel electrophoresis (PFGE). PFGE analysis of the 53 MRSA isolates revealed six major types (A to $\mathrm{F}$ ) and 25 subtypes. The B-type DNA pattern was indistinguishable from that of the South American epidemic clone observed in $34 \%$ of the isolates. A novel highly prevalent clone, showing the A-type DNA pattern and representing $38 \%$ of the isolates, was also identified. Moreover, the most frequent subtype of the A clonal family triggered an outbreak in a hospital 2 months later, further confirming its epidemic feature.

FROM: Sola C, Gribaudo G, Vindel A, Patrito L, Bocco JL. Identification of a novel methicillin-resistant Staphylococcus aureus epidemic clone in Cordoba, Argentina, involved in nosocomial infections. J Clin Microbiol 2002;40:1427-1435. 